\title{
Erratum: Neutrino charge radii from COHERENT elastic neutrino-nucleus scattering [Phys. Rev. D 98, 113010 (2018)]
}

\author{
M. Cadeddu, C. Giunti@, K. A. Kouzakov, Y. F. Li, A. I. Studenikin, and Y. Y. Zhang
}

(Received 26 February 2020; accepted 27 February 2020; published 16 March 2020)

DOI: 10.1103/PhysRevD.101.059902

In this erratum we revise the results given in the paper concerning the limits on the neutrino charge radii obtained from the analysis of the COHERENT data, that were affected by an incorrect treatment of the antineutrino contribution. In the paper we took into account the fact that neutrinos and antineutrinos have opposite charge radii, but we did not notice that also the weak neutral current couplings change sign from neutrinos to antineutrinos. Indeed, both the electromagnetic vector current and the $V-A$ weak neutral current change sign under a $C P$ transformation that changes left-handed neutrinos into righthanded antineutrinos. Therefore the relative sign of the weak neutral current and charge radius contributions does not change from neutrinos to antineutrinos. This correction leads to significant changes of the limits on the neutrino charge radii obtained from the analysis of the COHERENT data.

A revised version of our paper with the correct results and discussion is available as the last version of arXiv:1810.05606. Here we present: 1 ) the corrected version of Table II that contains the numerical results of the analysis of the COHERENT data, 2) the corrected version of Fig. 2 that illustrates the effects of the diagonal charge radii, and 3) the corrected versions of Figs. 3 and 6 that show the allowed regions in the $\left\langle r_{\nu_{e}}^{2}\right\rangle-\left\langle r_{\nu_{\mu}}^{2}\right\rangle$ plane. The corrections to the text of the paper are as follows:

(i) In the abstract the words "especially that of $\nu_{\mu}$ " must be removed.

(ii) Equation (18) and the surrounding text "This is important for the diagonal charge radii that contribute coherently with weak interactions in the cross section (14). Since ...[Eq. (18)]...neutrinos and antineutrinos contribute with different signs to the shift of $\sin ^{2} \vartheta_{W}$ in Eq. (1)." must be replaced with "However, also $g_{V}^{p}$ and $g_{V}^{n}$ change sign for antineutrinos. Therefore, the diagonal charge radii of neutrinos and antineutrinos generate the same shift of $\sin ^{2} \vartheta_{W}$ in Eq. (1)".

(iii) The words "whereas the allowed range of $\left\langle r_{\nu_{\mu}}^{2}\right\rangle$ increases by about $30 \%$ in the free $R_{n}$ approach" at the end of the text in page 6 must be removed.

(iv) Also the following paragraph "The authors of Ref. [17] obtained bounds on $\left\langle r_{\nu_{e}}^{2}\right\rangle$ and $\left\langle r_{\nu_{\mu}}^{2}\right\rangle$ from an analysis of the total number of COHERENT events. Their results are different from ours, after taking into account the factor-of-two different definitions of the neutrino charge radii discussed in Sec. II. It is likely that this is due to the assumption in

TABLE II. Results of the fits of the COHERENT data. The limits on the charge radii are at $90 \%$ C.L. and in units of $10^{-32} \mathrm{~cm}^{2}$.

\begin{tabular}{|c|c|c|c|c|c|c|c|c|}
\hline & \multicolumn{4}{|c|}{ Spectrum } & \multicolumn{4}{|c|}{ Spectrum and time } \\
\hline & \multicolumn{2}{|c|}{$\left\langle r_{\nu_{e}}^{2}\right\rangle$ and $\left\langle r_{\nu_{\mu}}^{2}\right\rangle$ only } & \multicolumn{2}{|c|}{ All $\left\langle r_{\nu}^{2}\right\rangle$} & \multicolumn{2}{|c|}{$\left\langle r_{\nu_{e}}^{2}\right\rangle$ and $\left\langle r_{\nu_{\mu}}^{2}\right\rangle$ only } & \multicolumn{2}{|c|}{ All $\left\langle r_{\nu}^{2}\right\rangle$} \\
\hline & Fixed $R_{n}$ & Free $R_{n}$ & Fixed $R_{n}$ & Free $R_{n}$ & Fixed $R_{n}$ & Free $R_{n}$ & Fixed $R_{n}$ & Free $R_{n}$ \\
\hline$\overline{\chi_{\min }^{2}}$ & 2.6 & 2.6 & 2.6 & 2.6 & 153.9 & 153.7 & 154.0 & 153.8 \\
\hline $\mathrm{NDF}$ & 142 & 140 & 139 & 137 & 142 & 140 & 139 & 137 \\
\hline GoF & $100 \%$ & $100 \%$ & $100 \%$ & $100 \%$ & $23 \%$ & $20 \%$ & $18 \%$ & $15 \%$ \\
\hline$\left\langle r_{\nu_{s}}^{2}\right\rangle$ & $(-76,27)$ & $(-77,32)$ & $(-74,27)$ & $(-73,29)$ & $(-56,6)$ & $(-56,8)$ & $(-56,6)$ & $(-55,6)$ \\
\hline$\left\langle r_{\nu}^{2}\right\rangle$ & $(-63,14)$ & $(-64,17)$ & $(-62,13)$ & $(-61,15)$ & $(-61,10)$ & $(-60,10)$ & $(-60,9)$ & $(-59,9)$ \\
\hline$\left|\left\langle r_{\nu_{e \mu}}^{2}\right\rangle\right|$ & & & $<31$ & $<31$ & & & $<28$ & $<28$ \\
\hline$\left|\left\langle r_{\nu_{e \tau}}^{2}\right\rangle\right|$ & & & $<51$ & $<51$ & & & $<31$ & $<30$ \\
\hline$\left|\left\langle r_{\nu_{\mu \tau}}^{2}\right\rangle\right|$ & & & $<38$ & $<38$ & & & $<34$ & $<35$ \\
\hline
\end{tabular}

Published by the American Physical Society under the terms of the Creative Commons Attribution 4.0 International license. Further distribution of this work must maintain attribution to the author(s) and the published articles title, journal citation, and DOI. 


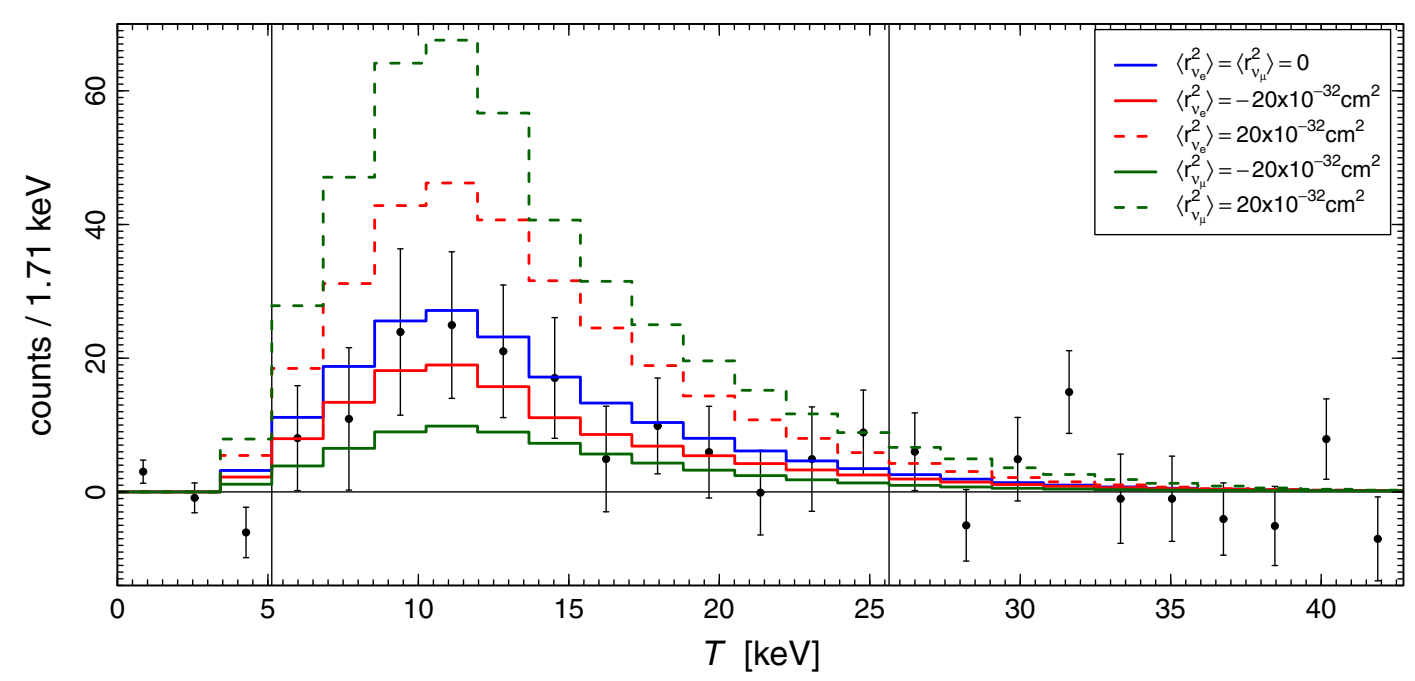

FIG. 2. COHERENT data [18,19] versus the nuclear kinetic recoil energy $T$. The blue histogram shows the theoretical predictions without neutrino charge radii and the theoretical values of the rms radii of the neutron distributions in Eqs. (24) and (25). The red and green histograms show the theoretical predictions obtained by adding the contributions of the neutrino charge radii reported in the legend. The two vertical lines at 5.13 and $25.64 \mathrm{keV}$ indicate the range of our analysis, as explained after Eq. (26).

Ref. [17] of $\left\langle r_{\bar{\nu}_{\ell}}^{2}\right\rangle=\left\langle r_{\nu_{\ell}}^{2}\right\rangle$, contrary to the correct relation in Eq. (18). Indeed, we checked that making that assumption the fit of the total number of COHERENT events yields results similar to those presented in Ref. [17]." at the beginning of the text in page 7 must be removed.

(v) The words "although the contours in the $\left\langle r_{\nu_{e}}^{2}\right\rangle-\left\langle r_{\nu_{\mu}}^{2}\right\rangle$ plane change shape" at the end of the following paragraph in page 7 must be replaced with "but the allowed region in the $\left\langle r_{\nu_{e}}^{2}\right\rangle-\left\langle r_{\nu_{\mu}}^{2}\right\rangle$ plane becomes simply connected. The hole in the allowed region with the diagonal charge radii alone is due to a cancellation between the weak interaction and charge radii contributions to the cross section. The hole disappears when the neutrino transition charge radii are taken into account, because their contribution to the cross section can fit the data."

(vi) The words "correspond to large negative values of $\left\langle r_{\nu_{e}}^{2}\right\rangle$ and very small values of $\left\langle r_{\nu_{\mu}}^{2}\right\rangle$ " in the last paragraph in page 7 must be replaced with "correspond to a large negative value of $\left\langle r_{\nu_{e}}^{2}\right\rangle$ in the left panel obtained without the transition charge radii, and large negative values of both $\left\langle r_{\nu_{e}}^{2}\right\rangle$ and $\left\langle r_{\nu_{\mu}}^{2}\right\rangle$ in the right panel obtained with the transition charge radii."

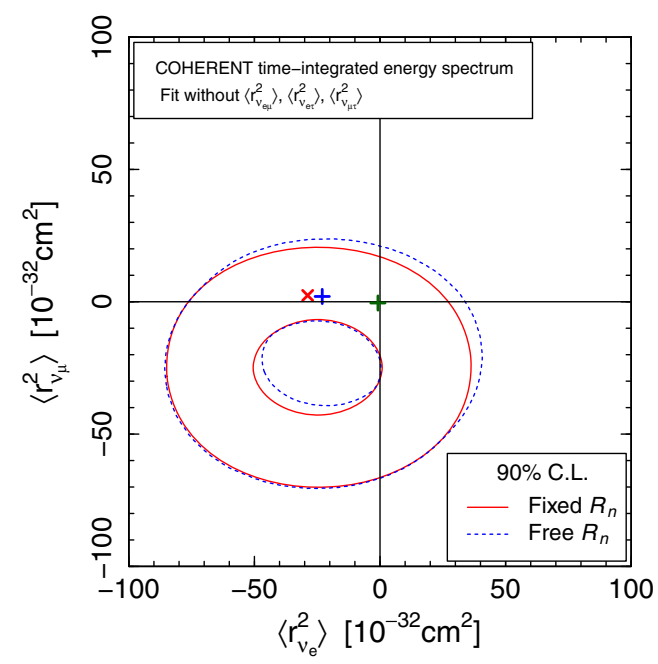

(a)

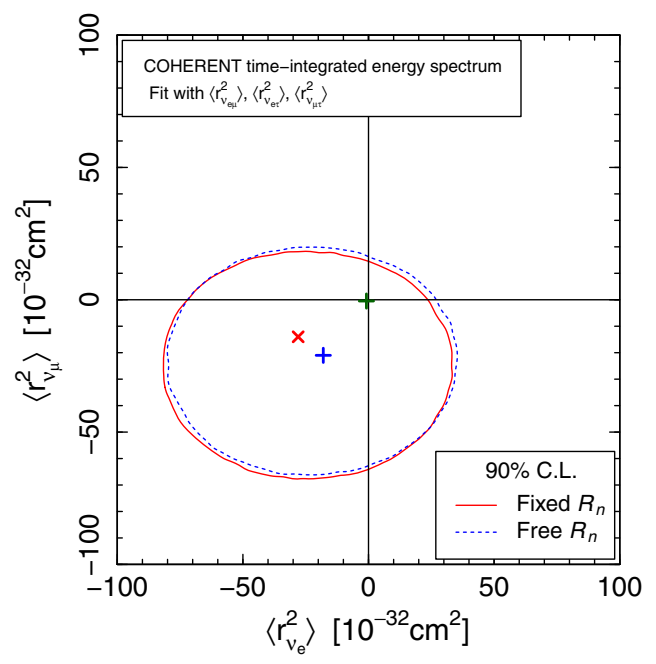

(b)

FIG. 3. $90 \%$ C.L. allowed regions in the $\left\langle r_{\nu_{e}}^{2}\right\rangle-\left\langle r_{\nu_{\mu}}^{2}\right\rangle$ plane obtained from the fit of the time-integrated COHERENT energy spectrum without (a) and with (b) the transition charge radii. The red and blue points indicate the best-fit values. The green point near the origin indicates the Standard Model values in Eqs. (5) and (6). 


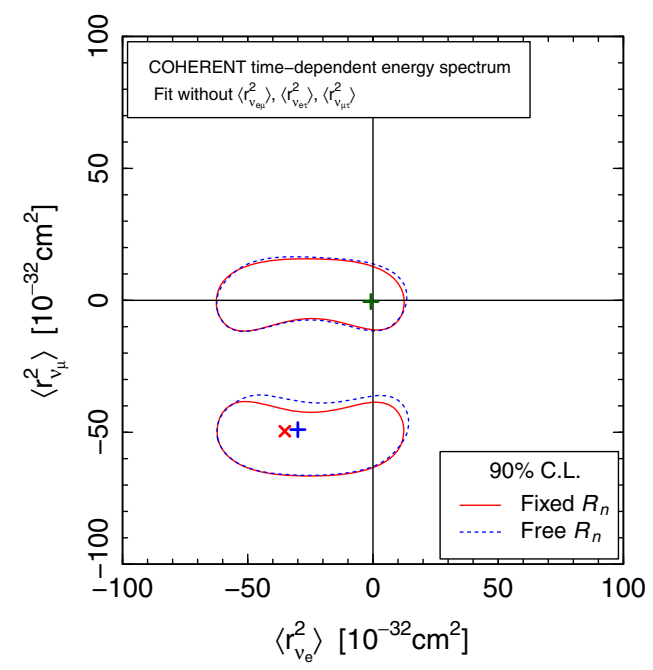

(a)

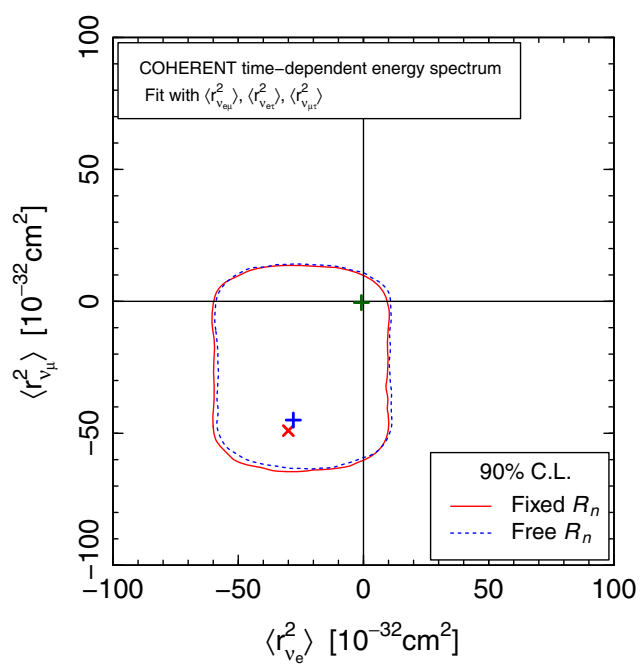

(b)

FIG. 6. $90 \%$ C.L. allowed regions in the $\left\langle r_{\nu_{e}}^{2}\right\rangle-\left\langle r_{\nu_{\mu}}^{2}\right\rangle$ plane obtained from the fit of the time-dependent COHERENT energy spectrum without (a) and with (b) the transition charge radii. The red and blue points indicate the best-fit values. The green point near the origin indicates the Standard Model values in Eqs. (5) and (6).

(vii) The words "especially $\left\langle r_{\nu_{\mu}}^{2}\right\rangle$. This is due to the difference of the prompt time distribution of $\nu_{\mu}$ 's and the delayed time distribution of $\bar{\nu}_{\mu}$ 's shown in Fig. 5, that severely constrains $\left\langle r_{\nu_{\mu}}^{2}\right\rangle$ through the relation (18). Therefore, the limits that we obtained $\left\langle r_{\nu_{\mu}}^{2}\right\rangle$ are comparable with those obtained in the BNL-E734 [47] and CHARM-II [48] experiments (see Table I)" in the first paragraph in page 9 must be removed.

(viii) The words "the best-fit values of $\left\langle r_{\nu_{e}}^{2}\right\rangle-\left\langle r_{\nu_{\mu}}^{2}\right\rangle$ shown by points in Fig. 6 correspond to large negative values of $\left\langle r_{\nu_{e}}^{2}\right\rangle$ and very small values of $\left\langle r_{\nu_{\mu}}^{2}\right\rangle$ " in the last paragraph of Sec. IV must be replaced by "the best-fit values of $\left\langle r_{\nu_{e}}^{2}\right\rangle$ and $\left\langle r_{\nu_{\mu}}^{2}\right\rangle$ shown by points in Fig. 6 correspond to large negative values of $\left\langle r_{\nu_{e}}^{2}\right\rangle$ and $\left\langle r_{\nu_{\mu}}^{2}\right\rangle$."

(ix) Equation (32) and the surrounding text "especially that of $\left\langle r_{\nu_{\mu}}^{2}\right\rangle$, for which we obtained the 90\% C.L. allowed interval ...[Eq. (32)]... marginalizing over reliable allowed intervals of the rms radii of the neutron distributions of ${ }^{133} \mathrm{Cs}$ and ${ }^{127}$ I. This limit is comparable with the BNL-E734 [47] and CHARM-II [48] limits in Tab. I" in Sec. V must be removed.

(x) The limits in Eq. (33) must be replaced with $(28,30,35) \times 10^{-32} \mathrm{~cm}^{2}$. 